\title{
Can the arts promote more humane health professionals? Exploring evidence from the neurosciences
}

\author{
Saroj Jayasinghe \\ Professor of Medicine, Faculty of Medicine, University of Colombo
}

The Sri Lankan health profession is in urgent need of being more humane, compassionate and empathic. This requires education to widen its scope than medical ethics and communication skills. Medical Humanities is one such interdisciplinary fields that draws on the creative and intellectual strengths of diverse disciplines related to the humanities, including, but not limited to philosophy, ethics, literature, art, creative writing, drama, film, music, anthropology, and history. The challenge is the use of these diverse fields in a creative manner to achieve the goal of developing more humane health professionals.

There are several approaches to describe the role of medical humanities and concepts such as humaneness, compassion and empathy. Two commonly used approaches are to explore their philosophical basis, and the underlying psychological principles. Another emerging area of interest is to study the neuro-scientific basis of humaneness (including compassion and empathy) and how these could be enhanced using a range of techniques.

It is well known that compassion, empathy and sympathy have biological, social and psychological roots. According to evolutionary psychology, the biological roots of altruism (a concept close to compassion) can be traced to advantages in survival and propagation of a species. Altruism attracts more

\section{Author responsible for correspondence:}

Saroj Jayasinghe, MBBS, MD (Col), FRCP (Lond), FCCP, MD (Bristol), $\mathrm{PhD}(\mathrm{Col})$

Email: saroj@clinmed.cmb.ac.lk

Current affiliations: Professor of Medicine and Head,

Department of Medical Humanities, University of

Colombo, Consultant Physician, National Hospital of

Sri Lanka

Phone - 0094718619331

http://orcid.org/0000-0003-1460-6073

DOI: http://doi.org/10.4038/cjms.v55i1.4938 partners, and promotes self-sacrifice to enable more members of a group to survive. This behaviour is observed in herds of wild buffaloes crossing crocodile infested rivers, when one lead animal self sacrificially creates a diversion to enable others to cross. There is emerging evidence from neuroscience that our brains are structurally networked to be altruistic and functional MRIs show that rewards of money, food, or sex all activate the same mesolimbic area of the brain (1).

Extremely altruistic individuals (e.g. kidney donors) have functional MRI characteristics that are inverse to those who are psychopaths (2). Volunteers making charitable donations over their own interests showed activity in a different area, e.g. the sub-genual cortex/ septal region which is closely related to social attachment and bonding. These experiments suggest that altruism is not due to suppression of selfishness, but a basic hard-wired feature of the brain that is pleasurable. There is also evidence that both, empathy, and the experience of the emotion one has empathy for, recruit similar networks of neurones (3).

Studies on the link between the arts and the neurosciences have led to the emerging field of neuroaesthetics which uses knowledge and techniques of neuroscience to describe and understand the aesthetic experiences at a neurological level. Still in its infancy, in relative terms, these studies have used EEGs, functional MRIs, and other forms of imaging to explain the visual features (e.g. symmetry, colour combinations, contrast, clarity, and complex nature of the image) and preferences for art and art appreciation (4). Some of these techniques enable perception and its judgement to be described according to their ophthalmological and neurological effects. For example, the smile in the portrait of Mona Lisa (by Leanardo da Vinci, 1452-1519) is considered emotionally ambiguous. It could be a happy smile or imply a tinge of sadness. The basis of this ambiguity is believed to be due to the lack of a distinct outline for 
the edges of the mouth, the gradation of hues used, or a more holistic processing by the brain of all the visual cues (5).

In contrast, abstract art has a different affect than representational art. They challenge the nervous system to scan and process images and visual information that are not compatible with the images that we are familiar with in nature. Famous examples include paintings of cubists (e.g. Pablo Picasso) who has distorted the 3-dimensional images and viewed the same object from several perspectives (6).

This intersection of art, cognitive science and philosophy has led to the emergence of niche areas for research, and examples include philosophical aesthetics and neuroaesthetics - the biological basis of aesthetic evaluations (7). The field has expanded to the domain of music in explaining the neuroscience of musical improvisation and interpretation of classical work.

Analogous to the scientific exploration of the arts, could we understand the neuroscience of humaneness and develop more objective interventions that would promote it? Is there evidence from neuroscience that suggests a link between the appreciation of the arts and humaneness? For example, would the humanities have an impact on the development of mirror neurones that could play a role in compassion? These questions are yet to be answered. However, the indications are that the arts could have an impact on mental health, emotions, mental disorders and recovery from acquired brain injury (8).

Though still lacking hard evidence, one could speculate that humanities would have similar positive influences in health professional education. Even if such evidence is not available, shouldn't we make these courses at least more interesting and enjoyable with an infusion of the arts?

\section{References}

1. Human fronto-mesolimbic networks guide decisions about charitable donation Proceedings of the National Academy of Sciences of the USA. 2006;103(42):15623-1562. https://doi.org/10.1073/pnas.0604475103

2. Abigail A, Marsh AA, Sarah A, Stoycos SA, Brethel-Haurwitz KM et al. Neural and cognitive characteristics of extraordinary altruists. Proceedings of the National Academy of Sciences of the USA. 2014; (42): 15036-15041. https://doi.org/10.1073/pnas.1408440111
3. Singer T, Lamm C. The social neuroscience of empathy. Annals of the New York Academy of Science 2009;1156; 81-96. doi:10.1111/j.1749-6632.2009.04418.x

4. Chatterjee Anjan. Neuroaesthetics: a coming of age story. Journal of Cognitive Neuroscience. 2011; 23 (1): 53-62. doi:10.1162/jocn.2010.21457

5. Bohrn I, Carbon CC, Hutzler F. Psychological Science 2010;21:378-380. doi: $10.1177 / 0956797610362192$

6. Reductionism in Art and Brain Science-Bridging the Two Cultures. Erica R Kandel. Colombia University Press, New York. 2016). doi: 10.1162/LEON_r_01438

7. Meskin A, Robson J, Ichino A, et al. Philosophical aesthetics and cognitive science. Wiley Interdisciplinary Reviews. Cognitive Science. 2018;9(1):1-15. doi: $10.1002 /$ wcs. 1445

8. Hegde S. Music therapy for mental disorder and mental health: the untapped potential of Indian classical music. British Journal of Psychiatry International. 2017;14(2):31-33. 